\title{
Alkynes as Privileged Synthons in Selected Organic Name Reactions
}

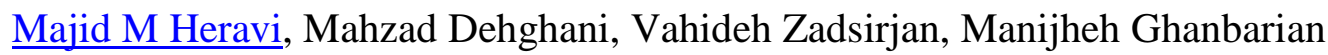

Department of Chemistry, School of Science, Alzahra University, Vanak, Tehran, Iran

\begin{abstract}
Background: Alkynes are actually basic chemicals, serving as privileged synthons for planning new organic reactions for assemblage of a reactive motif, which easily undergoes a further desirable transformation. Name reactions, in organic chemistry are referred to those reactions which are well-recognized and reached to such status for being called as their explorers, discoverers or developers. Alkynes have been used in various name reactions. In this review, we try to underscore the applications of alkynes as privileged synthons in prevalent name reactions such as Huisgen 1,3-dipolar cycloaddtion via Click reaction, Sonogashira reaction, and Hetero Diels-Alder reaction.

Objective: In this review, we try to underscore the applications of alkynes as privileged synthons in the formation of heterocycles, focused on the selected reactions of alkynes as a synthon or impending utilization in synthetic organic chemistry, which have reached such high status for being included in the list of name reactions in organic chemistry.
\end{abstract}

Conclusion: Alkynes (including acetylene) are an unsaturated hydrocarbon bearing one or more triple C-C bond. Remarkably, alkynes and their derivatives are frequently being used as molecular scaffolds for planning new organic 
Source: Heravi MM, Dehghani M, Zadsirjan V, Ghanbarian M. Alkynes as privileged synthons in selected organic name reactions. Curr Org Synth. 2019;16(2):205-43.

reactions and installing reactive functional group for further reaction. It is worth mentioning that in general, the terminal alkynes are more useful and more frequently being used in the art of organic synthesis. Remarkably, alkynes have found different applications in pharmacology, nanotechnology, as well as being known as appropriate starting precursors for the total synthesis of natural products and biologically active complex compounds. They are predominantly applied in various name reactions such as Sonogashira, Glaser reaction, Friedelcrafts reaction, Castro-Stephens coupling, Huisgen 1.3-dipolar cycloaddtion reaction via Click reaction, Sonogashira reaction, hetero-Diels-Alder reaction. In this review, we tried to impress the readers by presenting selected name reactions, which use the alkynes as either stating materials or precursors. We disclosed the applications of alkynes as a privileged synthons in several popular reactions, which reached to such high status being classified as name reactions. They are thriving and well known and established name reactions in organic chemistry such as Regioselective, 1,3-dipolar Huisgen cycloaddtion reaction via Click reaction, Sonogashira reaction and Diels-Alder reaction.

Keywords: 3-dipolar cycloaddtion; Alkynes; click reaction; hetero-diels-alder reaction; heterocycles; huisgen 1; sonogashira reaction. 